\title{
Predictors of pathological complete response to neoadjuvant treatment and changes to post-neoadjuvant HER2 status in HER2-positive invasive breast cancer
}

\author{
Ayaka Katayama $\mathbb{1}^{1,2} \cdot$ Islam M. Miligy ${ }^{1,3} \cdot$ Sho Shiino ${ }^{1,4} \cdot$ Michael S. Toss $\mathbb{1}^{1} \cdot$ Karim Eldib $^{5} \cdot$ Sasagu Kurozumi ${ }^{6,7}$. \\ Cecily M. Quinn ${ }^{8} \cdot$ Nahla Badr $^{3,9} \cdot$ Ciara Murray $^{8}$ - Elena Provenzano ${ }^{10} \cdot$ Grace Callagy $^{11} \cdot$ Cian Martyn $^{11}$. \\ Rebecca Millican-Slater ${ }^{12}$. Colin Purdie $\mathbb{1}^{13} \cdot$ Dave Purnell $^{14} \cdot$ Sarah E. Pinder $\mathbb{( D}^{15} \cdot$ Tetsunari Oyama $^{2}$. \\ Abeer M. Shaaban ${ }^{9} \cdot \operatorname{lan}$ Ellis $\mathbb{1}^{1,5} \cdot$ Andrew H. S. Lee ${ }^{5} \cdot$ Emad A. Rakha $^{1,5}$
}

Received: 26 October 2020 / Revised: 21 December 2020 / Accepted: 22 December 2020 / Published online: 1 February 2021

(c) The Author(s) 2021. This article is published with open access

\begin{abstract}
The response of human epidermal growth factor receptor2 (HER2)- positive breast cancer (BC) patients to anti-HER2 targeted therapy is significant. However, the response is not uniform and a proportion of HER2-positive patients do not respond. This study aims to identify predictors of response in the neoadjuvant treatment and to assess the discordance rate of HER2 status between pre- and post-treatment specimens in HER2-positive BC patients. The study group comprised $500 \mathrm{BC}$ patients treated with neoadjuvant chemotherapy (NACT) and/or neoadjuvant anti-HER2 therapy and surgery who had tumours that were $3+$ or $2+$ with HER2 immunohistochemistry (IHC). HER2 IHC $2+$ tumours were classified into five groups by fluorescence in situ hybridisation (FISH) according to the 2018 ASCO/CAP guidelines of which Groups 1, 2 and 3 were considered HER2 amplified. Pathological complete response (pCR) was more frequent in HER2 IHC $3+$ tumours than in HER2 IHC 2+/HER2 amplified tumours, when either in receipt of NACT alone (38\% versus $13 \% ; p=0.22$ ) or neoadjuvant anti-HER2 therapy ( $52 \%$ versus $20 \% ; p<0.001)$. Multivariate logistic regression analysis showed that HER2 IHC $3+$ and histological grade 3 were independent predictors of pCR following neoadjuvant anti-HER2 therapy. In the HER2 IHC 2+/HER2 amplified tumours or ASCO/CAP FISH Group 1 alone, ER-negativity was an independent predictor of pCR following NACT and/or neoadjuvant anti-HER2 therapy. In the current study, 22\% of HER2-positive tumours became HER2-negative by IHC and FISH following neoadjuvant treatment, the majority (74\%) HER2 IHC 2+/HER2 amplified tumours. Repeat HER2 testing after neoadjuvant treatment should therefore be considered.
\end{abstract}

\section{Introduction}

Approximately $15 \%$ of invasive breast cancers (BCs) are human epidermal growth factor receptor2 (HER2) positive, defined as showing HER2 gene amplification or protein overexpression, and such tumours have been shown to be sensitive to anti-HER2 targeted therapy [1-3]. Currently, a combination of sequential chemotherapy and anti-HER2

These authors contributed equally: Ayaka Katayama, Islam M. Miligy

Emad A. Rakha

emad.rakha@nottingham.ac.uk

Extended author information available on the last page of the article therapy is the standard treatment for HER2-positive BC both in the neoadjuvant and adjuvant setting [4]. Immunohistochemistry (IHC) and/or in situ hybridisation (ISH) is routinely used to evaluate the HER2 status for treatment selection.

The current American Society of Clinical Oncology/ College of American Pathologists (ASCO/CAP) guidelines regard HER2 IHC score $3+$ as positive, score $2+$ as equivocal and scores 0 and $1+$ as negative [5]. If the IHC result is score 2+, such patients are tested for HER2 amplification by ISH; most commonly fluorescence in situ hybridisation (FISH), to assess the average HER2 gene and chromosome enumeration probe 17 (CEP17) copy numbers (CNs) per carcinoma cell and the ratio of these [5]. The 2018 ASCO/ CAP guidelines divide HER2 FISH status into five groups (Table 1) [5]. 
Table 1 HER2 FISH groups in 2018 ASCO/CAP guidelines.

\begin{tabular}{lll}
\hline Group & HER2/CEP17 ratio & Average HER2 gene CN \\
\hline 1 & $\geq 2.0$ & $\geq 4.0$ \\
2 & $\geq 2.0$ & $<4.0$ \\
3 & $<2.0$ & $\geq 6.0$ \\
4 & $<2.0$ & $\geq 4.0$ and $<6.0$ \\
5 & $<2.0$ & $<4.0$ \\
\hline
\end{tabular}

$C N$ copy number.

For HER2-positive BC, defined as either IHC $3+$ or IHC $2+$ with HER2 amplification, neoadjuvant chemotherapy (NACT) plus neoadjuvant anti-HER2 therapy is an effective treatment option [4]. The pathological complete response (pCR) rate at the time of surgery is commonly used as an endpoint in clinical trials and a predictor of good prognosis in HER2-positive BC with neoadjuvant treatment [6-8]. The most widely-agreed definition of $\mathrm{pCR}$ is no residual invasive carcinoma both in the breast and axillary lymph nodes regardless of the presence of residual ductal carcinoma in situ (DCIS) (ypT0/is ypN0) [8].

Retrospective studies of HER2-positive BC patients who have received NACT plus neoadjuvant anti-HER2 therapy have reported a higher rate of $\mathrm{pCR}$ in IHC $3+$ compared to IHC 2+/HER 2 amplified tumours [9]. However, little attention has been paid to predictors of pCR among the different ASCO/CAP FISH groups. In addition, some clinical trials showed that $\mathrm{pCR}$ rates are lower in oestrogen receptor (ER)-positive/HER2-positive BC than in ERnegative/HER2-positive BC [10-12]. It remains unclear if this holds true for the different ASCO/CAP FISH amplified groups.

Meanwhile, others studies have reported discordant HER2 status between the pre-treatment biopsy and the posttreatment surgical specimen [13-18]. Loss of HER2positivity in the residual tumour has been reported to be associated with a poorer outcome compared to tumours that remain HER2-positive following neoadjuvant treatment [16-18]. The change in HER2 status may also affect the selection of post-neoadjuvant treatment. However, there is no consensus on whether or not repeat HER2 testing in post-neoadjuvant residual disease should be performed in routine practice.

In this study, we have analysed 500 invasive BCs with HER2 IHC $3+$, or IHC 2+ for which HER2 FISH data were available, from patients who received NACT and/or neoadjuvant anti-HER2 therapy with subsequent therapeutic surgery. Our aim was to evaluate the relationship between HER2 categories and pCR alongside other variables. We also report the level of concordance for $H E R 2$ and the ASCO/CAP FISH groups between the pre- and posttreatment specimens in this study cohort.

\section{Materials and methods}

\section{Study cohort}

A total of 500 invasive BCs with HER2 IHC $3+$ or IHC $2+$ for which HER2 FISH data were available from patients who received NACT and/or neoadjuvant anti-HER2 therapy with subsequent therapeutic surgery between 2013 and 2020 were included. Exclusion criteria were: (1) no information of HER2 gene $\mathrm{CN}$ or HER2/CEP17 ratio in pretreatment specimens for IHC $2+$ tumours; (2) patients treated with neoadjuvant hormonal therapy alone; (3) lack of data on pathological response in the surgical specimen. Therefore 75 of the 575 patients were excluded. The majority of patients were treated at Nottingham University Hospitals NHS Trust, Nottingham $(n=254)$ with additional patients from the following nine institutions: Addenbrookes Hospital, Cambridge; University Hospitals Birmingham NHS Foundation Trust; University Hospitals of Leicester NHS Trust; St. Vincent's University Hospital, Dublin; University Hospital Galway, Galway; Burney Breast Unit, St Helens and Knowsley Teaching Hospital NHS Trust, Liverpool; Guy's and St Thomas' NHS Foundation Trust, London; Ninewells Hospital, Dundee; University of Turin, Turin Italy. Patients with tumours that demonstrated an HER2 IHC score of $2+$ but that were non-amplified with FISH were included as a control group $(n=151)$.

Patients were considered eligible for anti-HER2 therapies if their tumours showed a HER2 IHC score of $3+$, or $2+$ with a ratio $\geq 2.0$ regardless of the HER2 $\mathrm{CN}$ or if the HER2 gene $\mathrm{CN}$ was $\geq 6$, as recommended by UK guidelines (corresponding to ASCO/CAP FISH Groups 1, 2 and 3) [19]. Treatment was given according to institutional guidelines. Exact neoadjuvant regimens and number of cycles varied slightly but patients were divided into four groups according to the neoadjuvant treatment received: chemotherapy alone; chemotherapy with trastuzumab; chemotherapy with dual anti-HER2 agents (i.e. trastuzumab with either pertuzumab or lapatinib) and anti-HER2 therapy alone.

pCR was defined as no residual invasive carcinoma in both breast and axillary lymph nodes regardless of the presence of residual DCIS (ypT0/is ypN0) [8]. Histological grade was evaluated according to the Nottingham modification of the Scarff-Bloom-Richardson system on the pretreatment specimens [20]. All of the histopathological data used in the analysis were derived from the original pathology reports.

\section{Immunohistochemistry and FISH assay}

IHC for ER and progesterone receptor (PR), and both HER2 IHC and FISH for HER2 in pre- and post-treatment 
specimens were assessed as per UK guidelines [19, 21]. HER2 IHC was scored as positive (3+), equivocal $(2+)$ or negative $(1+/ 0)$, and IHC score $2+$ patients were tested for HER2 amplification by FISH [19]. As per the 2018 ASCO/CAP guidelines, HER2 FISH status was assigned to one of five groups (Table 1) [5]. However, it was impossible to completely follow the recommendation for concomitant IHC review and reassessment and recounting of FISH slides in the less common FISH patterns (Groups 2, 3 and 4) owing to the retrospective nature of this study. In this study, ASCO/CAP FISH Groups 1, 2 and 3 were defined as $H E R 2$ amplified according to UK guidelines, which differ from 2018 ASCO/CAP guidelines where Group 2 tumours are now considered non-amplified [19]. According to CEP17 CN, chromosome 17 (chr 17) status was defined as monosomy of chr17 (m17) if $<1.5$, normal chr17 (n17) if $\geq 1.5$ but $<3.0$, and polysomy of chr17 (p17) if $\geq 3.0$ average $\mathrm{CN}$ per carcinoma cells [22, 23]. For ER and PR, tumours were classified as positive when there was $\geq 1 \%$ nuclear staining in invasive carcinoma cells [21].

\section{Statistical analysis}

Statistical analysis was performed using EZR (Saitama Medical Center Jichi Medical University; http://www.jichi. ac.jp/saitama-sct/SaitamaHP.files/statmed.html), which is a graphical user interface for R (The R Foundation for Statistical Computing, Vienna, Austria, version 2.13.0) [24]. Associations between clinicopathological variables and pCR were examined with Fisher's exact tests or Pearson's $\chi^{2}$ test, as appropriate. A logistic regression model was applied to evaluate the effect of covariates on pCR. If a variable remained at a level of $p$ value $\leq 0.15$, it was incorporated into the final multivariable model [25]. Comparison between pre- and post-treatment receptor status was assessed by McNemar's test. A $p$ value $\leq 0.05$ was considered statistically significant. This study was approved by the Nottingham Research Tissue Bank Access Committee under the IRAS Project ID: 184265. Data collected were fully anonymised.

\section{Results}

\section{Patient characteristics}

Table 2 shows the demographic and treatment characteristics of the study cohort by HER 2 categories. The median age at diagnosis was similar amongst all the groups. The tumour histological type was most commonly invasive breast carcinoma of no special type (NST), whilst histological grade was predominantly 2 or 3 across the whole cohort. ER and PR were both more often negative in IHC 3+ tumours than in other groups $(p<0.001)$. As expected, patients with HER2-positive tumours were significantly more likely to receive anti-HER2 therapy $(p<0.001)$. Chemotherapy with trastuzumab was the main neoadjuvant treatment in patients with tumours that were HER2 IHC $3+$ or IHC $2+$ within the HER2 amplified group (ASCO/CAP FISH Groups 1, 2 and 3), while all patients within the HER2 non-amplified group (Groups 4 and 5) received chemotherapy alone. An anthracycline- and taxane-based chemotherapy regimen was received by the majority of patients across all groups, but the number of patients treated without an anthracycline regimen was significantly higher in Group 1 $(p<0.001)$.

\section{HER2 categories and pathologic complete response}

Comparisons of pCR rate within the HER2 categories were made according to ER and PR status and whether antiHER2 therapy was given (Fig. 1). For whole patients, relationship between treatment regimens and pCR rate among various HER2 categories was different (Fig. 1A). For IHC $3+$ tumours, the pCR rate was $37.5 \%(n=6 / 16)$ for patients treated with chemotherapy alone and $51.8 \%$ (85/164) following anti-HER2 therapy. Among IHC $2+$ tumours, the $\mathrm{pCR}$ rate following chemotherapy alone was as follows: $12.5 \%$ (2/16) in the HER2 amplified group (Groups 1,2 and 3); $12.5 \%(1 / 8)$ in Group $1 ; 16.7 \%(1 / 6)$ in Group 2; $0 \%(0 / 2)$ in Group 3; $12.6 \%(19 / 151)$ in the HER2 nonamplified group (Groups 4 and 5). The pCR rate in IHC $2+$ tumours when anti-HER2 therapy was also given was as follows: $20.3 \%$ (31/153) in HER2 amplified tumours (Groups 1, 2 and 3); 20.2\% (21/104) in Group 1; 21.4\% (9/ 42 ) in Group 2; and $14.3 \%$ (1/7) in Group 3. Thus, IHC 3+ tumours had higher rates of pCR than IHC $2+/$ HER 2 amplified tumours, when either in receipt of chemotherapy alone $(37.5 \%$ versus $12.5 \% ; p=0.22)$ or anti-HER 2 therapy $(51.8 \%$ versus $20.3 \% ; p<0.001)$.

We then evaluated the likelihood of pCR following different anti-HER2 therapies (chemotherapy plus single anti-HER2 therapy or chemotherapy plus dual anti-HER2 therapy) for HER2-positive tumours (Fig. 1B). Whether treated with chemotherapy plus single or chemotherapy plus dual anti-HER2 therapy, patients with IHC $3+$ tumours had a significantly higher $\mathrm{pCR}$ rate than those with the IHC $2+/ H E R 2$ amplified tumours (pCR rate in chemo+anti-HER2-single, $50.0 \%$ in IHC $3+$ versus $19.4 \%$ in IHC $2+/$ HER 2 amplified; $p<0.001$ ) (pCR rate in chemo+anti-HER2-dual, 59.3\% in IHC $3+$ versus $17.6 \%$ in IHC $2+/$ HER 2 amplified; $p=0.01$ ). For IHC $3+$ tumours, chemotherapy plus dual anti-HER2 therapy showed higher $\mathrm{pCR}$ rate than chemotherapy plus single anti-HER2 therapy $(59.3 \%$ versus $50.0 \% ; p=0.41)$. 
Table 2 Patients baseline characteristics.

\begin{tabular}{|c|c|c|c|c|c|c|c|}
\hline \multirow[t]{2}{*}{ Characteristic } & \multirow{2}{*}{$\begin{array}{l}\text { IHC3+ }(n= \\
180) \text { No. }(\%)\end{array}$} & \multicolumn{5}{|c|}{ IHC $2+(n=320)$} & \multirow[t]{2}{*}{$P$ value } \\
\hline & & $\begin{array}{l}\text { Group } 1(n= \\
112) \text { No. }(\%)\end{array}$ & $\begin{array}{l}\text { Group } 2(n= \\
48) \text { No. }(\%)\end{array}$ & $\begin{array}{l}\text { Group } 3(n=9) \\
\text { No. }(\%)\end{array}$ & $\begin{array}{l}\text { Group } 4(n= \\
55) \text { No. }(\%)\end{array}$ & $\begin{array}{l}\text { Group } 5(n= \\
\text { 96) No. }(\%)\end{array}$ & \\
\hline \multicolumn{8}{|l|}{ Age } \\
\hline Median [range] & 52 [23-83] & $52[27-86]$ & $52[27-78]$ & $50[36-71]$ & 54 [23-73] & 53 [26-75] & \\
\hline \multicolumn{8}{|l|}{ Histology type } \\
\hline Ductal, NST & $160(88.9)$ & 97 (86.6) & $43(89.6)$ & $9(100)$ & $48(87.3)$ & $83(86.5)$ & 0.45 \\
\hline Special types & $7(3.9)$ & $8(7.1)$ & $2(4.2)$ & 0 & $4(7.3)$ & $1(1.0)$ & \\
\hline Lobular & $6(3.3)$ & $6(5.4)$ & $2(4.2)$ & 0 & $3(5.4)$ & $9(9.4)$ & \\
\hline $\begin{array}{l}\text { Mixed (ductal and } \\
\text { lobular) }\end{array}$ & $4(2.2)$ & $1(0.9)$ & 0 & 0 & 0 & $3(3.1)$ & \\
\hline Unknown & $3(1.7)$ & 0 & $1(2.0)$ & 0 & 0 & 0 & \\
\hline \multicolumn{8}{|l|}{ Histological grade } \\
\hline 1 & $2(1.1)$ & $1(0.9)$ & $1(2.1)$ & 0 & $1(1.8)$ & $2(2.1)$ & 0.17 \\
\hline 2 & $103(57.2)$ & $55(49.1)$ & $26(54.2)$ & $3(33.3)$ & $25(45.5)$ & $64(66.7)$ & \\
\hline 3 & $64(35.6)$ & $52(46.4)$ & $20(41.6)$ & $6(66.7)$ & $29(52.7)$ & $28(29.1)$ & \\
\hline Unknown & $11(6.1)$ & $4(3.6)$ & $1(2.1)$ & 0 & 0 & $2(2.1)$ & \\
\hline \multicolumn{8}{|l|}{ ER } \\
\hline Positive & $103(57.2)$ & $87(77.7)$ & $35(72.9)$ & $9(100)$ & $44(80.0)$ & $72(75.0)$ & $<0.001$ \\
\hline Negative & $75(41.7)$ & $25(22.3)$ & $13(27.1)$ & 0 & $11(20.0)$ & $24(25.0)$ & \\
\hline Unknown & $2(1.1)$ & 0 & 0 & & & & \\
\hline \multicolumn{8}{|l|}{$\mathrm{PR}$} \\
\hline Positive & $69(38.3)$ & $51(45.5)$ & $21(43.8)$ & $7(77.8)$ & $28(50.9)$ & $53(55.2)$ & $<0.001$ \\
\hline Negative & $110(61.1)$ & $37(33.1)$ & $15(31.2)$ & $2(22.2)$ & $15(27.3)$ & $36(37.5)$ & \\
\hline Unknown & $1(0.6)$ & $24(21.4)$ & $12(25.0)$ & 0 & $12(21.8)$ & $7(7.3)$ & \\
\hline \multicolumn{8}{|l|}{ Neoadjuvant treatments } \\
\hline Chemotherapy alone & $16(8.9)$ & $8(7.1)$ & $6(12.5)$ & $2(22.2)$ & $55(100)$ & $96(100)$ & $<0.001$ \\
\hline $\begin{array}{l}\text { Chemotherapy }+ \text { anti- } \\
\text { HER2 therapy (single) }\end{array}$ & $134(74.4)$ & $87(77.7)$ & $37(77.1)$ & $5(55.6)$ & 0 & 0 & \\
\hline $\begin{array}{l}\text { Chemotherapy +dual anti- } \\
\text { HER2 therapy }\end{array}$ & $27(15.0)$ & 13 (11.6) & $3(6.2)$ & $1(11.1)$ & 0 & 0 & \\
\hline Anti-HER2 therapy alone & $3(1.7)$ & $4(3.6)$ & $2(4.2)$ & $1(11.1)$ & 0 & 0 & \\
\hline \multicolumn{8}{|l|}{ Chemotherapy regimens } \\
\hline $\begin{array}{l}\text { Anthracyclines and } \\
\text { Taxanes }\end{array}$ & $128(72.3)$ & $66(61.1)$ & $36(78.3)$ & $5(62.5)$ & $44(80.0)$ & $75(78.1)$ & $<0.001$ \\
\hline $\begin{array}{l}\text { Anthracyclines without } \\
\text { Taxanes }\end{array}$ & $32(18.1)$ & $6(5.6)$ & $2(4.3)$ & $1(12.5)$ & $6(10.9)$ & $17(17.7)$ & \\
\hline Non-anthracyclines & $17(9.6)$ & $36(33.3)$ & $8(17.4)$ & $2(25.0)$ & $5(9.1)$ & $4(4.2)$ & \\
\hline
\end{tabular}

According to 2018 ASCO/CAP guidelines, HER2 FISH status were divided into five groups in IHC $2+$ patients: group 1, HER2/CEP17 ratio $\geq$ 2.0, average HER2 gene $\mathrm{CN} \geq 4.0$; group 2, HER2/CEP17 ratio $\geq 2.0$, HER2 gene $\mathrm{CN}<4.0$; group 3, HER2/CEP17 ratio $<2.0$, HER2 gene CN $>$ 6.0; group 4, HER2/CEP17 ratio $<2.0$, average HER2 gene $\mathrm{CN} \geq 4.0$ and $\leq 6.0$; and group 5, HER2/CEP17 ratio $<2.0$, HER2 gene CN $<4.0$.

Bold values indicate statistical significance $p<0.05$.

IHC immunohistochemistry, NST no special type.

For patients who received anti-HER2 therapy (Fig. 1C, D), those who had ER-negative tumours showed a significantly higher pCR rate than those who had ER-positive tumours in Group $1(p=0.01)$. PR status did not significantly affect the pCR rate.

\section{Prediction of pCR according to HER2 categories}

The association between clinicopathological and treatment parameters and the attainment of a pCR was examined in the whole cohort, in the different HER2 categories and in 
A
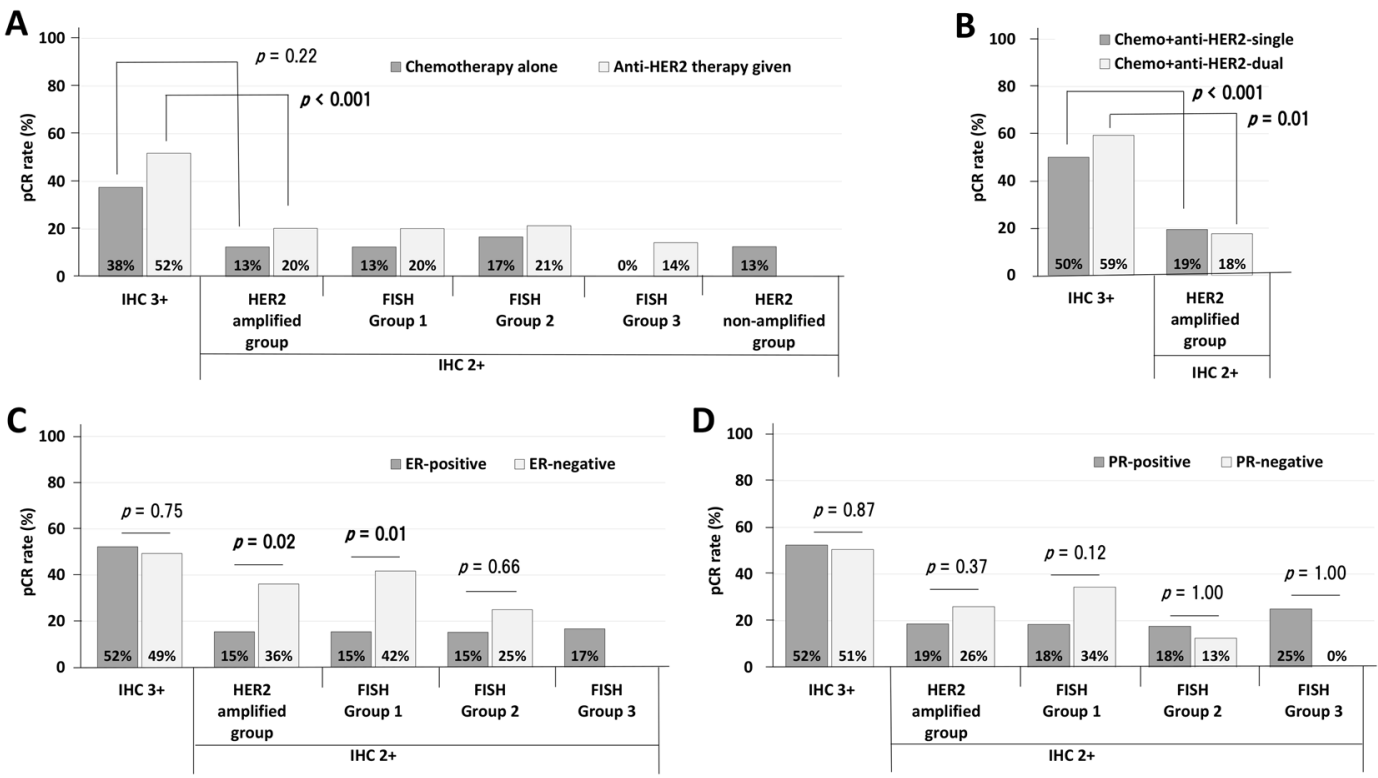

Fig. 1 The pathological complete response rate in HER2 categories. (A) according to types of neoadjuvant treatment for whole patients, (B) different anti-HER2 therapies for HER2-positive patients, (C) ER status for anti-HER2 therapy given patients, and (D) PR status for anti-HER2 therapy given patients.

those who received anti-HER2 therapy by univariate and multivariate stepwise regression model (Table 3). Within the cohort of patients with IHC $3+$ tumours, pCR was not associated with any additional factor studied. Among those with IHC 2+/HER2 amplified tumours (Groups 1, 2 and 3), ER-negativity was identified as an independent predictor of pCR (ER negative versus positive; OR, 11.80; 95\%CI, $1.38-101.00 ; p=0.02$ ) which remained the case in Group 1 alone (ER negative versus positive; OR, 3.71; 95\% CI, $1.28-10.70 ; p=0.02$ ). In Group 2 , histological grade 3 was an independent predictor of pCR (3 versus 1, 2; OR, 7.86; $95 \%$ CI, $1.39-44.40 ; p=0.02$ ). For patients treated with anti-HER2 therapy, histological grade 3 (3 versus 1, 2; OR, $1.750 ; 95 \% \mathrm{CI}, 1.02-3.01 ; p=0.04)$ and HER2 IHC $3+$ (IHC $3+$ versus IHC 2+/HER2 amplified; OR, 3.490; 95\% CI, 1.98-6.16; $p<0.001$ ) were identified as independent predictors of $\mathrm{pCR}$.

\section{Changes in ER, PR and HER2 status after neoadjuvant treatment}

HER2 status was assessed in the residual invasive carcinoma present after NACT and/or neoadjuvant anti-HER2 therapy in 221 patients. For comparison any ER and PR status changes were also assessed (143, 140 patients respectively) (Table 4). Of the 139 patients with pretreatment HER2-positive tumours (IHC $3+$ or IHC $2+1$ HER2 amplified), 31 tumours (22.3\%) become HER2negative (IHC $2+/ H E R 2$ non-amplified, or IHC $0 / 1+$ ) after treatment, whereas 13/82 (15.9\%) of patients with HER2negative tumours before treatment changed to HER2- positive following treatment $(p=0.01)$. In our cohort, neoadjuvant treatment regimens were not significantly associated with changes to post-neoadjuvant HER2 categories (data not shown). None of the 101 ER-positive tumours before treatment changed to ER-negative after treatment, whereas $4 / 42(9.5 \%)$ of patients with pretreatment ER-negative tumours changed to ER-positive. Of the 76 patients with PR-positive tumours before treatment, 15 (19.7\%) had PR-negative tumours after treatment, whereas 11/64 (17.2\%) patients with PR-negative tumours before treatment changed to PR-positive. These differences were not significant $(p>0.05)$.

We then evaluated HER2 concordance between pre- and post-treatment specimens according to the different HER2 categories (Table 5). The highest level of concordance (78.4\%) was observed for IHC3 + tumours followed by $55.2 \%$ in ASCO/CAP FISH Group 2. No tumours within ASCO/CAP FISH Groups 1, 2 or 3 in the pre-treatment specimen were assessed IHC $3+$ in the post-treatment excision specimen. Focussing on the 5 ASCO/CAP FISH groups, 62 patients $(45.6 \%)$ showed a change in the FISH group pre- and post-treatment; $9.6 \%$ of HER2 amplified tumours (Groups 1, 2 and 3) changed FISH group whilst, $16.9 \%$ of HER 2 non-amplified tumours (Groups 4 and 5) changed. The ASCO/CAP FISH group was unchanged in $54.4 \%$ of patients. Importantly, $38.1 \%$ (16/42) of tumours that were identified as being within Group 1 in the pretreatment specimen changed to HER 2 non-amplified group in the excision specimen.

Focusing on the average HER2 gene and CEP17 CNs, we evaluated HER2 discordance between pre- and post- 
Table 3 Univariate and multivariate logistic regression model for pCR according to HER2 categories or anti-HER2 therapy given.

\begin{tabular}{|c|c|c|c|c|c|c|c|c|c|}
\hline \multirow[t]{2}{*}{ Parameters } & \multirow[t]{2}{*}{ Risk/reference } & \multicolumn{5}{|c|}{ Univariant analysis } & \multicolumn{3}{|c|}{ Multivariant analysis } \\
\hline & & $\begin{array}{l}\mathrm{pCR} / \text { non-pCR } \\
\text { No. }(\%)\end{array}$ & $\begin{array}{l}\mathrm{pCR} / \text { non-pCR } \\
\text { No. }(\%)\end{array}$ & OR & $95 \% \mathrm{CI}$ & $P$ value & OR & $95 \% \mathrm{CI}$ & $P$ value \\
\hline \multicolumn{10}{|l|}{ IHC $3+$} \\
\hline Age & $\geq 50 /<50$ & $47 / 54(46.5)$ & $42 / 34(49.4)$ & 0.71 & $0.39-1.28$ & 0.29 & - & - & - \\
\hline Histological grade & $3 / 1,2$ & $36 / 28(56.2)$ & $47 / 58(62.7)$ & 1.59 & $0.85-2.97$ & 0.16 & - & - & - \\
\hline ER & Negative/positive & $38 / 37(50.6)$ & $51 / 52(49.5)$ & 1.05 & $0.58-1.90$ & 1 & - & - & - \\
\hline PR & Negative/positive & $57 / 53(51.8)$ & $33 / 36(47.8)$ & 1.17 & $0.64-2.14$ & 0.65 & - & - & - \\
\hline Anti-HER2 therapy & Yes/No & $85 / 79(51.8)$ & $6 / 10(37.5)$ & 1.79 & $0.62-5.16$ & 0.31 & - & - & - \\
\hline $\begin{array}{l}\text { Chemotherapy } \\
\text { regimens }\end{array}$ & $\begin{array}{l}\text { Anthracyclines/Non- } \\
\text { anthracyclines }\end{array}$ & $81 / 79(50.6)$ & $8 / 9(47.1)$ & 1.15 & $0.42-3.14$ & 0.80 & - & - & - \\
\hline \multicolumn{10}{|c|}{ HER2 amplified group (Groups 1, 2 and 3) } \\
\hline Age & $\geq 50 /<50$ & 19/81 (19.0) & $14 / 54(20.6)$ & 0.91 & $0.42-1.96$ & 0.85 & - & - & - \\
\hline Histological grade & $3 / 1,2$ & $23 / 55(29.5)$ & $10 / 76(11.6)$ & 3.18 & $1.40-7.21$ & 0.006 & 2.10 & $0.83-5.36$ & 0.12 \\
\hline ER & Negative/positive & $15 / 23(39.5)$ & $18 / 113(13.7)$ & 4.09 & $1.81-9.29$ & $<0.001$ & 11.80 & $1.38-101.00$ & 0.02 \\
\hline PR & Negative/positive & $15 / 39(27.8)$ & $13 / 66(16.5)$ & 1.95 & $0.84-4.53$ & 0.133 & 0.252 & $0.03-2.07$ & 0.20 \\
\hline Anti-HER2 therapy & Yes/No & $31 / 122(20.3)$ & $2 / 14(12.5)$ & 1.78 & $0.38-8.24$ & 0.74 & - & - & - \\
\hline $\begin{array}{l}\text { Chemotherapy } \\
\text { regimens }\end{array}$ & $\begin{array}{l}\text { Anthracyclines/Non- } \\
\text { anthracyclines }\end{array}$ & 20/96 (17.2) & $10 / 36(21.7)$ & 0.75 & $0.32-1.76$ & 0.51 & - & - & - \\
\hline \multicolumn{10}{|l|}{ FISH Group 1} \\
\hline Age & $\geq 50 /<50$ & $14 / 52(21.2)$ & 8/38 (17.4) & 1.28 & $0.49-3.35$ & 0.81 & - & - & - \\
\hline Histological grade & $3 / 1,2$ & $14 / 38(26.9)$ & $8 / 48(14.3)$ & 2.21 & $0.84-5.82$ & 0.15 & 1.58 & $0.56-4.45$ & 0.39 \\
\hline ER & Negative/positive & $10 / 15(40.0)$ & $12 / 75(13.8)$ & 4.17 & $1.52-11.40$ & 0.008 & 3.71 & $1.28-10.70$ & 0.02 \\
\hline PR & Negative/positive & $11 / 26(29.7)$ & $9 / 42(17.6)$ & 1.97 & $0.72-5.41$ & 0.21 & - & - & - \\
\hline Anti-HER 2 therapy & Yes/No & $21 / 83(20.2)$ & $1 / 7(12.5)$ & 1.77 & $0.21-15.20$ & 0.60 & - & - & - \\
\hline $\begin{array}{l}\text { Chemotherapy } \\
\text { regimens }\end{array}$ & $\begin{array}{l}\text { Anthracyclines/Non- } \\
\text { anthracyclines }\end{array}$ & $12 / 60(16.7)$ & $9 / 27(25.0)$ & 0.6 & $0.23-1.59$ & 0.31 & - & - & - \\
\hline \multicolumn{10}{|l|}{ FISH Group 2} \\
\hline Age & $\geq 50 /<50$ & $4 / 25(13.8)$ & 6/13 (31.6) & 0.35 & $0.08-1.45$ & 0.16 & - & - & - \\
\hline Histological grade & $3 / 1,2$ & $8 / 12(40.0)$ & $2 / 25(7.4)$ & 8.33 & $1.53-45.40$ & 0.01 & 7.86 & $1.39-44.40$ & 0.02 \\
\hline ER & Negative/positive & $5 / 8(38.5)$ & $5 / 30(14.3)$ & 3.75 & $0.87-16.20$ & 0.11 & 3.27 & $0.66-16.30$ & 0.15 \\
\hline PR & Negative/positive & 4/11 (26.7) & $3 / 18(14.3)$ & 2.18 & $0.41-11.60$ & 0.42 & - & - & - \\
\hline Anti-HER2 therapy & Yes/No & $9 / 33(21.4)$ & $1 / 5(16.7)$ & 1.36 & $0.14-13.20$ & 1.00 & - & - & - \\
\hline $\begin{array}{l}\text { Chemotherapy } \\
\text { regimens }\end{array}$ & $\begin{array}{l}\text { Anthracyclines/Non- } \\
\text { anthracyclines }\end{array}$ & $7 / 31(18.4)$ & $1 / 7(12.5)$ & 1.58 & $0.17-15.00$ & 1.00 & - & - & - \\
\hline \multicolumn{10}{|c|}{ Anti-HER2 therapy given } \\
\hline Age & $\geq 50 /<50$ & $59 / 121(32.8)$ & $55 / 78(41.4)$ & 0.69 & $0.43-1.10$ & 0.12 & 0.71 & $0.42-1.22$ & 0.22 \\
\hline Histological grade & $3 / 1,2$ & $55 / 78(41.4)$ & $53 / 116(31.4)$ & 1.54 & $0.96-2.48$ & 0.09 & 1.75 & $1.02-3.01$ & 0.04 \\
\hline ER & Negative/positive & $47 / 57(45.2)$ & $67 / 144(31.8)$ & 1.77 & $1.09-2.87$ & 0.02 & 1.34 & $0.64-2.82$ & 0.43 \\
\hline PR & Negative/positive & $64 / 85(43.0)$ & $46 / 88(34.3)$ & 1.44 & $0.89-2.33$ & 0.15 & 1.07 & $0.52-2.21$ & 0.86 \\
\hline HER2 & $3+/ 2+$ & $85 / 79(51.8)$ & $31 / 122(20.3)$ & 4.23 & $2.57-6.98$ & $<0.001$ & 3.49 & $1.98-6.16$ & $<0.001$ \\
\hline $\begin{array}{l}\text { Chemotherapy } \\
\text { regimens }\end{array}$ & $\begin{array}{l}\text { Anthracyclines/Non- } \\
\text { anthracyclines }\end{array}$ & 93/152 (38.0) & $18 / 44(29.0)$ & 1.50 & $0.82-2.74$ & 0.24 & - & - & - \\
\hline
\end{tabular}

According to 2018 ASCO/CAP guidelines, HER2 FISH status were divided into five groups in IHC 2+ patients: group 1, HER2/CEP17 ratio $\geq 2.0$, average HER2 gene $\mathrm{CN} \geq 4.0$; group 2, HER2/CEP17 ratio $\geq 2.0, H E R 2$ gene $\mathrm{CN}<4.0$; group 3, HER2/CEP17 ratio $<2.0, H E R 2$ gene $\mathrm{CN}>6.0$; group 4, HER2/CEP17 ratio <2.0, average HER2 gene $\mathrm{CN} \geq 4.0$ and $<6.0$; and group 5, HER2/CEP17 ratio $<2.0, H E R 2$ gene $\mathrm{CN}<4.0$.

Bold values indicate statistical significance $p<0.05$.

$I H C$ immunohistochemistry, FISH fluorescence in situ hybridisation, $p C R$ pathological complete response, $O R$ odds ratio, $C I$ confidence interval. 
treatment specimens among the IHC $2+$ tumours (Table 6). Of the 23 patients that changed HER2 category from IHC $2+/ H E R 2$ amplified to IHC2+/HER 2 non-amplified, $15(65.2 \%)$ maintained the same HER2 gene $\mathrm{CN}$, whereas $8(34.8 \%)$ showed a decreased HER2 gene CN. In addition, chr 17 status was divided into $\mathrm{m} 17, \mathrm{n} 17$ and p17 according to CEP17 CN. Of the previously mentioned 23 patients, $17(73.9 \%)$ maintained the same chr 17 status, while $2(8.7 \%)$ patients with $\mathrm{n} 17$ before treatment changed to p17 after treatment. For comparison 13 patients that changed category from an IHC $2+/ H E R 2$ nonamplified to IHC2+/HER 2 amplified were assessed; of the 13 patients, $10(76.9 \%)$ maintained the same HER2 gene $\mathrm{CN}$, whereas $3(23.1 \%)$ showed an increased HER2 gene $\mathrm{CN}$. According to CEP17 $\mathrm{CN}$, of the 13 patients,

Table 4 Comparison of HER2, ER and PR status between the pretreatment core biopsy and the post-treatment excision specimen.

\begin{tabular}{|c|c|c|c|c|}
\hline \multirow[t]{2}{*}{ Pre-treatment } & \multirow[t]{2}{*}{ Total no. (\%) } & \multicolumn{2}{|c|}{ Post-treatment } & \multirow[t]{2}{*}{$P$ value } \\
\hline & & $\begin{array}{l}\text { Positive } \\
\text { no. }(\%)\end{array}$ & $\begin{array}{l}\text { Negative } \\
\text { no. }(\%)\end{array}$ & \\
\hline \multicolumn{5}{|l|}{ HER2 } \\
\hline Positive & 139 (62.9) & $108(77.7)$ & $31(22.3)$ & \multirow[t]{2}{*}{0.01} \\
\hline Negative & $82(37.1)$ & 13 (15.9) & $69(84.1)$ & \\
\hline \multicolumn{5}{|l|}{ ER } \\
\hline Positive & $101(70.6)$ & $101(100)$ & 0 & \multirow[t]{2}{*}{0.13} \\
\hline Negative & $42(29.4)$ & $4(9.5)$ & $38(90.5)$ & \\
\hline \multicolumn{5}{|l|}{$\mathrm{PR}$} \\
\hline Positive & $76(54.3)$ & $61(80.3)$ & $15(19.7)$ & \multirow[t]{2}{*}{0.56} \\
\hline Negative & $64(45.7)$ & $11(17.2)$ & $53(82.8)$ & \\
\hline
\end{tabular}

Bold values indicate statistical significance $p<0.05$.
$10(76.9 \%)$ maintained the same chr 17 status, while $1(7.7 \%)$ with $\mathrm{n} 17$ before treatment changed to $\mathrm{m} 17$.

\section{Discussion}

In the current study, we aimed to identify predictive factors for pCR after NACT across different HER2-positive categories. We showed that HER2 IHC 3+ invasive BC had a higher pCR rate than IHC $2+/ H E R 2$ amplified tumours when anti-HER2 therapy was received, consistent with earlier analyses [9]. In our study, pCR rate of HER2 IHC 3 + tumours following anti-HER2 therapy was $52 \%$, within the range reported in clinical trials with similar treatment regimens; for example, pCR rate was $38 \%$ in the NOAH trial using NACT with trastuzumab, 39\% in the NeoSphere and $58 \%$ in the TRYPHAENA trials using NACT with trastuzumab and pertuzumab $[10,26,27]$. In keeping with the significant impact on pCR that dual anti-HER2 therapy has shown [26-28], we also identified a higher pCR rate for NACT with dual anti-HER2 therapy compared to NACT with single ani-HER 2 therapy in patients with IHC $3+$ BC. Notably, the pCR rates among HER2 IHC 2+/HER2 amplified tumours in patients who received anti-HER2 therapy were $20 \%$ in Group 1, 21\% in Group 2 and $14 \%$ in Group 3, lower than the clinical trials. Regardless of ASCO/ CAP FISH groups, IHC 2+/HER2 amplified tumours showed significantly lower rates of pCR than IHC $3+$ tumours. Consistent with previous studies, our data highlight that HER2 IHC $3+$ and histological grade 3 are independent predictors of $\mathrm{pCR}$ following treatment with anti-HER2 therapy $[9,29]$. Although a higher rate of pCR in HER2 IHC 3+ tumours was reported in the

Table 5 Correlation between HER2 categories in pre-treatment core biopsy and the post-treatment excision specimen.

\begin{tabular}{|c|c|c|c|c|c|c|c|c|}
\hline \multirow{3}{*}{$\begin{array}{l}\text { Pre- } \\
\text { treatment }\end{array}$} & \multirow{3}{*}{$\begin{array}{l}\text { Total } \\
\text { no. }(\%)\end{array}$} & \multicolumn{7}{|c|}{ Post-treatment } \\
\hline & & \multirow{2}{*}{$\begin{array}{l}\text { IHC } 3+ \\
\text { No. }(\%)\end{array}$} & \multicolumn{5}{|l|}{ IHC $2+$} & \multirow[t]{2}{*}{ IHC $1+$ or 0} \\
\hline & & & $\begin{array}{l}\text { Group } 1 \\
\text { no. }(\%)\end{array}$ & $\begin{array}{l}\text { Group } 2 \\
\text { no. }(\%)\end{array}$ & $\begin{array}{l}\text { Group } 3 \\
\text { no. }(\%)\end{array}$ & $\begin{array}{l}\text { Group } 4 \\
\text { no. }(\%)\end{array}$ & $\begin{array}{l}\text { Group } 5 \\
\text { no. }(\%)\end{array}$ & \\
\hline IHC $3+$ & $65(29.4)$ & $51(78.4)$ & $4(6.2)$ & $2(3.0)$ & 0 & 0 & $4(6.2)$ & $4(6.2)$ \\
\hline \multicolumn{9}{|l|}{ IHC $2+$} \\
\hline Group 1 & $42(19.0)$ & 0 & $21(50.0)$ & $5(11.9)$ & 0 & $4(9.5)$ & $12(28.6)$ & 0 \\
\hline Group 2 & $29(13.1)$ & 0 & $7(24.1)$ & $16(55.2)$ & 0 & 0 & $6(20.7)$ & 0 \\
\hline Group 3 & $3(1.4)$ & 0 & $1(33.3)$ & 0 & $1(33.3)$ & $1(33.3)$ & 0 & 0 \\
\hline Group 4 & $27(12.2)$ & 0 & 4 (14.9) & $1(3.7)$ & 0 & $11(40.7)$ & $11(40.7)$ & 0 \\
\hline Group 5 & $55(24.9)$ & 0 & $5(9.1)$ & $3(5.4)$ & 0 & $2(3.6)$ & $25(45.5)$ & $20(36.4)$ \\
\hline
\end{tabular}

According to 2018 ASCO/CAP guidelines, HER2 FISH status were divided into five groups in IHC 2+ patients: group 1, HER2/CEP17 ratio $\geq$ 2.0, average HER2 gene $\mathrm{CN} \geq 4.0$; group 2, HER2/CEP17 ratio $\geq 2.0$, HER2 gene $\mathrm{CN}<4.0$; group 3, HER2/CEP17 ratio $<2.0$, HER2 gene CN $>$ 6.0; group 4, HER2/CEP17 ratio $<2.0$, average HER2 gene $\mathrm{CN} \geq 4.0$ and $\leq 6.0$; and group 5, HER2/CEP17 ratio $<2.0$, HER2 gene $\mathrm{CN}<4.0$.

Bold values indicate statistical significance $p<0.05$.

IHC immunohistochemistry. 
Table 6 Correlation between HER2 gene $\mathrm{CN}$ and CEP17 CN in pre-treatment core biopsy and the post-treatment excision specimen among HER2 discordant patients.

\begin{tabular}{|c|c|c|c|c|c|c|}
\hline \multirow{4}{*}{$\begin{array}{l}\text { Pre-treatment } \\
\text { HER2 gene CN }\end{array}$} & \multicolumn{6}{|l|}{ Post-treatment } \\
\hline & \multirow{2}{*}{\multicolumn{3}{|c|}{$\begin{array}{l}\text { HER2 discordance (HER2 amplified to non-amplified) } \\
(n=23) \\
\text { HER2 gene } \mathrm{CN}\end{array}$}} & \multirow{2}{*}{\multicolumn{3}{|c|}{$\begin{array}{l}\text { HER2 discordance (HER2 non-amplified to amplified) } \\
(n=13) \\
\text { HER2 gene } \mathrm{CN}\end{array}$}} \\
\hline & & & & & & \\
\hline & \multicolumn{2}{|l|}{$<6.0$ No. $(\%)$} & $\geq 6.0$ No. $(\%)$ & \multicolumn{2}{|l|}{$<6.0$ No. $(\%)$} & $\geq 6.0$ No. $(\%)$ \\
\hline$<6.0$ & \multicolumn{2}{|l|}{$15(65.2)$} & 0 & \multicolumn{2}{|l|}{$10(76.9)$} & $3(23.1)$ \\
\hline$\geq 6.0$ & \multicolumn{2}{|l|}{$8(34.8)$} & 0 & \multicolumn{2}{|l|}{0} & 0 \\
\hline \multirow[b]{2}{*}{ CEP17 CN } & \multicolumn{3}{|l|}{ CEP17 CN } & \multicolumn{3}{|l|}{ CEP17 CN } \\
\hline & $\begin{array}{l}\mathrm{m} 17(<1.5) \\
\text { No. }(\%)\end{array}$ & $\begin{array}{l}\mathrm{n} 17(\geq 1.5,<3.0) \\
\text { No. }(\%)\end{array}$ & $\begin{array}{l}\text { p17 }(\geq 3.0) \\
\text { No. }(\%)\end{array}$ & $\begin{array}{l}\mathrm{m} 17(<1.5) \\
\text { No. }(\%)\end{array}$ & $\begin{array}{l}\text { n17 }(\geq 1.5,<3.0) \\
\text { No. }(\%)\end{array}$ & $\begin{array}{l}\text { p17 }(\geq 3.0) \\
\text { No. }(\%)\end{array}$ \\
\hline $\mathrm{m} 17(<1.5)$ & $1(4.3)$ & $2(8.7)$ & 0 & $1(7.7)$ & $1(7.7)$ & 0 \\
\hline $\mathrm{n} 17(\geq 1.5,<3.0)$ & 0 & $15(65.2)$ & $2(8.7)$ & $1(7.7)$ & $9(69.2)$ & 0 \\
\hline $\mathrm{p} 17(\geq 3.0)$ & 0 & $2(8.7)$ & $1(4.3)$ & 0 & $1(7.7)$ & 0 \\
\hline
\end{tabular}

CN copy number, $m 17$ monosomy chromosome 17, n17 normal chromosome 17, p17 polysomy chromosome 17.

Krystel-Whittemore study [9], their cohort had higher proportions of histological grade 3, ER negative and patients who received dual anti-HER2 therapy. This is in keeping with our analysis of the HER2 IHC $3+$ tumours that confirmed that a higher pCR rate was seen in histological grade 3 tumours treated with NACT and dual antiHER2 therapy (64\%).

Overall, in the HER2 IHC 2+/HER2 amplified group treated with NACT and/or anti-HER2 therapy, ERnegativity was an independent predictor of pCR. Furthermore, we identified independent predictors of pCR by ASCO/CAP FISH group treated with NACT and/or antiHER2 therapy: ER-negativity in Group 1 and histological grade 3 in Group 2. Predictors were not seen in the very small numbers of patients with tumours in Group 3. Indeed, because Groups 2, 3 and 4 are uncommon, the response of tumours in the different ASCO/CAP FISH groups to NACT has not been well explored [30]. Further analyses using a larger cohort are needed to validate these results, however our findings provide preliminary evidence that predictors of pCR differ between the ASCO/CAP FISH groups.

Hormone receptor (HR) status influences the response of a tumour to chemotherapy and the sensitivity of a tumour to combined chemotherapy and anti-HER2 therapy also differs according to HR status in the neoadjuvant setting [9-12,31]. Because HER2-positive, HR-negative tumours are likely to be highly dependent on the HER 2 gene for growth, these tumours typically show a good response to anti-HER2 therapies [32]. In the present series, in patients who received anti-HER2 therapy, ER-negativity was significantly associated with pCR in univariate analysis, but was not an independent predictive factor in multivariate analysis. We observed that HER2 IHC 2+/HER2 amplified ER-negative tumours showed a significantly higher rate of pCR than HER2 amplified ER-positive tumours. Meanwhile, in HER2 IHC 3+ tumours neither ER-negative nor PR-negative subgroups had a significantly higher rate of $\mathrm{pCR}$, similar to the findings of Miolo et al [33]. It has been previously reported that pathological characteristics differ between HER2 IHC $3+$ and HER2 IHC 2+/HER2 amplified tumours: IHC $3+$ tend to be of higher histological grade, larger tumour size, and are often ER-negative and PR-negative [34]. These findings emphasise the importance of considering combined HER2 and HR status to select those patients most likely to benefit from neoadjuvant anti-HER2 therapy.

Despite lower rates of pCR in HER2-positive/HR-positive tumours, it has been reported that these tumours have a good prognosis, and that there is thus a weaker association between pCR and long-term outcome in HER2-positive/ HR-positive tumours than HER2-positive/HR-negative tumours $[8,35]$. However, neoadjuvant endocrine therapy for HER2-positive/HR-positive patients has not resulted in a marked improvement in pCR rate [35]. Further strategies are required to increase the $\mathrm{pCR}$ rate and improve outcome in patients with HER2-positive/HR-positive BC.

Our results again highlight variation in HER2, ER and PR status in some cancers before and after neoadjuvant treatment, although the reported frequency of this varies [13-18]. The changes in HER2 status with loss of HER2 expression in the post-treatment specimens was statistically significant. This may reflect the response of the HER2 positive clone in the tumour to NACT and/or anti-HER2 therapy, leaving the HER2 negative clone as a residual component. Similarly, the acquisition of HER 2 positivity post treatment in a few cases is likely to reflect heterogeneity of HER2 expression. Of note, however, there was less frequent change in HER2 status following 
Table 7 HER2-positive patient baseline characteristics between neoadjuvant chemotherapy alone and anti-HER2 therapy given.

\begin{tabular}{|c|c|c|c|}
\hline \multirow[t]{2}{*}{ Characteristic } & \multicolumn{2}{|c|}{ Neoadjuvant treatments } & \multirow[t]{2}{*}{$P$ valu } \\
\hline & $\begin{array}{l}\text { Chemotherapy } \\
\text { alone }(n=32) \\
\text { No. }(\%)\end{array}$ & $\begin{array}{l}\text { anti-HER2 } \\
\text { therapy given } \\
(n=317) \\
\text { No. }(\%)\end{array}$ & \\
\hline \multicolumn{4}{|l|}{ Age } \\
\hline Median [range] & $53[29-80]$ & $52[23-86]$ & \\
\hline \multicolumn{4}{|l|}{ Histology type } \\
\hline Ductal, NST & $24(75.0)$ & $285(89.9)$ & 0.04 \\
\hline Special types & $4(12.5)$ & $13(4.1)$ & \\
\hline Lobular & $3(9.4)$ & $11(3.5)$ & \\
\hline $\begin{array}{l}\text { Mixed (ductal } \\
\text { and lobular) }\end{array}$ & 0 & $5(1.6)$ & \\
\hline Unknown & $1(3.1)$ & $3(0.9)$ & \\
\hline \multicolumn{4}{|l|}{ Histological grade } \\
\hline 1 & $1(3.1)$ & $3(0.9)$ & 0.18 \\
\hline 2 & $21(65.7)$ & $166(52.4)$ & \\
\hline 3 & $9(28.1)$ & $133(42.0)$ & \\
\hline Unknown & $1(3.1)$ & $15(4.7)$ & \\
\hline \multicolumn{4}{|l|}{ ER } \\
\hline Positive & $23(71.9)$ & $211(66.6)$ & 0.69 \\
\hline Negative & $9(28.1)$ & $104(32.8)$ & \\
\hline Unknown & 0 & $2(0.6)$ & \\
\hline \multicolumn{4}{|l|}{ PR } \\
\hline Positive & $14(43.7)$ & $134(42.3)$ & 1 \\
\hline Negative & $15(46.9)$ & $149(47.0)$ & \\
\hline Unknown & $3(9.4)$ & $34(10.7)$ & \\
\hline
\end{tabular}

Bold values indicate statistical significance $p<0.05$.

NST no special type.

neoadjuvant NACT and/or anti-HER2 therapy in cases defined as definitely positive by IHC (HER2 IHC $3+$ ) on pre-treatment specimens, compared to those that were IHC $2+$. It is unclear whether loss of HER2 amplification reflects response to therapy, a mechanism of resistance or heterogeneity of HER2 expression [14]. If such changes in HER2 status will affect post-neoadjuvant treatment decisions, such as the tailoring of subsequent adjuvant therapy, based on these results, we suggest that HER2 should be re-tested in post-neoadjuvant surgical specimens, particularly in HER2 2+/HER2 amplified tumours. Moreover, it has been reported that such alterations provide prognostic information with loss of HER2-positivity in residual tumours after neoadjuvant treatment shown to be associated with a poorer outcome compared with tumours with preserved HER2-positive status [16-18].

Our study has some limitations. First, this was a retrospective non-randomised study, and our samples were collected from multiple institutions which may have some selection bias effect. Second, some subset analyses were underpowered to detect subgroup differences due to small sample size, especially those in HER2 2+/FISH Group 3 BC $(n=9)$. Third, because of differences in regimens of chemotherapy prescribed, the influence of those on the different HER2-positive groups of $\mathrm{BC}$ with respect to $\mathrm{pCR}$ needs further study. Finally, not all patients with HER2-positive tumours received anti-HER2 therapy in this cohort; some patients, including some older women with co-morbidities and those with small and node-negative HER2-positive tumours, were less likely to receive neoadjuvant anti-HER2 therapy. In our cohort, there were no significantly differences in clinicopathological features such as age, HR status and histological grade whether anti-HER2 therapy given or not (Table 7). Special tumour type was a high percentage in patients received chemotherapy alone $(p=0.04)$.

In conclusion, the data presented here indicate that the maximum benefit of neoadjuvant anti-HER2 therapy is observed in the subgroup of patients with tumours that are HER 2 IHC $3+$, histological grade 3 or IHC $2+/$ HER2 amplification co-existing with ER-negativity. Among tumours that were HER2 IHC 2+/HER2 amplified, the predictors of $\mathrm{pCR}$ were different in the various ASCO/CAP FISH groups. In our study, 22\% of HER2-positive tumours before treatment changed to HER2-negative after neoadjuvant treatment, more commonly in HER2 IHC 2+/HER2 amplified tumours, especially ASCO/CAP FISH Group 1. Reassessment of HER2 status following neoadjuvant treatment should be considered in patients in whom it will facilitate further management decisions.

Acknowledgements We acknowledge all those who contributed to the collection of patients and to the Nottingham Health Science Biobank for supporting this study. AMS is supported by the Birmingham CRUK Centre (C17422/A25154). We thank Dr Michael Neat (Division of Cancer Studies, King's College London, Guy's Hospital, London, UK) for excellent technical assistance.

\section{Compliance with ethical standards}

Conflict of interest The authors declare that they have no conflict of interest.

Publisher's note Springer Nature remains neutral with regard to jurisdictional claims in published maps and institutional affiliations.

Open Access This article is licensed under a Creative Commons Attribution 4.0 International License, which permits use, sharing, adaptation, distribution and reproduction in any medium or format, as long as you give appropriate credit to the original author(s) and the source, provide a link to the Creative Commons license, and indicate if changes were made. The images or other third party material in this article are included in the article's Creative Commons license, unless indicated otherwise in a credit line to the material. If material is not included in the article's Creative Commons license and your intended use is not permitted by statutory regulation or exceeds the permitted use, you will need to obtain permission directly from the copyright holder. To view a copy of this license, visit http://creativecommons. org/licenses/by/4.0/. 


\section{References}

1. Dodson A, Parry S, Ibrahim M, Bartlett JM, Pinder S, Dowsett M, et al. Breast cancer biomarkers in clinical testing: analysis of a UK national external quality assessment scheme for immunocytochemistry and in situ hybridisation database containing results from 199300 patients. J Pathol Clin Res. 2018;4:262-73.

2. Giordano SH, Temin S, Kirshner J, Chandarlapaty S, Crews JR, Davidson NE, et al. Systemic therapy for patients with advanced human epidermal growth factor receptor 2-positive breast cancer: American Society of Clinical Oncology clinical practice guideline. J Clin Oncol. 2014;32:2078-99.

3. Loibl A, Gianni L. HER2-positive breast cancer. Lancet. 2017;389:2415-29.

4. Burstein HJ, Curigliano G, Loibl S, Dubsky P, Gnant M, Poortmans P, et al. Estimating the benefits of therapy for early-stage breast cancer: the St. Gallen international consensus guidelines for the primary therapy of early breast cancer 2019. Ann Oncol. 2019;30:1541-57.

5. Wolff AC, Hammond MEH, Allison KH, Harvey B, Mangu PB, Bartlett JMS, et al. Human epidermal growth factor receptor 2 testing in breast cancer: American Society of Clinical Oncology/ College of American Pathologists clinical practice guideline focused update. J Clin Oncol. 2018;36:2105-22.

6. von Minckwitz G, Untch M, Blohmer JU, Costa SD, Eidtmann H, Fasching PA, et al. Definition and impact of pathologic complete response on prognosis after neoadjuvant chemotherapy in various intrinsic breast cancer subtypes. J Clin Oncol. 2012;30:1796-804.

7. Kim MM, Allen P, Gonzalez-Angulo AM, Woodward WA, Meric-Bernstam F, Buzdar AU, et al. Pathologic complete response to neoadjuvant chemotherapy with trastuzumab predicts for improved survival in women with HER2-overexpressing breast cancer. Ann Oncol. 2013;24:1999-2004.

8. Cortazar P, Zhang L, Untch M, Mehta K, Costantino JP, Wolmark $\mathrm{N}$, et al. Pathological complete response and long-term clinical benefit in breast cancer: the CTNeoBC pooled analysis. Lancet. 2014;384:164-72.

9. Krystel-Whittemore M, Xu J, Brogi E, Ventura K, Patil S, Ross DS, et al. Pathologic complete response rate according to HER2 detection methods in HER2-positive breast cancer treated with neoadjuvant systemic therapy. Breast Cancer Res Treat. 2019;177:61-6.

10. Schneeweiss A, Chia S, Hickish T, Harvey V, Eniu A, Hegg R, et al. Pertuzumab plus trastuzumab in combination with standard neoadjuvant anthracycline-cintaining and anthracycline-free chemotherapy regimens in patients with HER2-positive early breast cancer: a randomized phase II cardiac safety study (TPYPHAENA). Ann Oncol. 2013;24:2278-84.

11. Hurvitz SA, Martin M, Symmans WF, Jung KH, Huang CS, Thompson AM, et al. Neoadjuvant trastuzumab, pertuzumab, and chemotherapy versus trastuzumab emtansine plus pertuzumab in patients with HER2-positive breast cancer (KRISTINE): a randomized, open-label, multicentre, phase 3 trial. Lancet Oncol. 2018;19:115-26.

12. Swain SM, Ewer MS, Viale G, Delaloge S, Ferrero JM, Verrill M, et al. Pertuzumab, trastuzumab, and standard anthracycline- and taxane- based chemotherapy for neoadjuvant treatment of patients with HER2-positive localized breast cancer (BERENICE): a phase II, open-label, multicenter, multinational cardiac safety study. Ann Oncol. 2018;29:646-53.

13. Li P, Liu T, Wang Y, Shao S, Zhang W, Lv Y, et al. Influence of neoadjuvant chemotherapy on HER2/neu status in invasive breast cancer. Clin Breast Cancer. 2013;13:53-60.

14. Niikura N, Tomotaki A, Miyata H, Iwamoto T, Kawai M, Anan $\mathrm{K}$, et al. Changes in tumor expression of HER2 and hormone receptors status after neoadjuvant chemotherapy in 21,755 patients from the Japanese breast cancer registry. Ann Oncol. 2016;27:480-7.

15. Gahlaut R, Bennett A, Fatayer H, Dall BJ, Sharma N, Velikova G, et al. Effect of neoadjuvant chemotherapy on breast cancer phenotype, ER/PR and HER2 expression - Implications for the practising oncologist. Eur J Cancer. 2016;60:40-8.

16. Mittendorf EA, Wu Y, Scaltriti M, Meric-Bernstam F, Hunt KK, Dawood $S$, et al. Loss of HER2 amplification following trastuzumab-based neoadjuvant systemic therapy and survival outcomes. Clin Cancer Res. 2009;15:7381-8.

17. Guarneri V, Dieci MV, Barbieri E, Piacentini F, Omarini C, Ficarra $G$, et al. Loss of HER2 positivity and prognosis after neoadjuvant therapy in HER2-positive breast cancer patients. Ann Oncol. 2013;24:2990-4.

18. Branco FP, Machado D, Silva FF, André S, Catarino A, Madureira R, et al. Loss of HER2 and disease prognosis after neoadjuvant treatment of HER2 + breast cancer. Am J Transl Res. 2019;11:6110-6.

19. Rakha EA, Pinder SE, Bartlett JM, Ibrahim M, Starczynski J, Carder PJ, et al. Updated UK Recommendations for HER2 assessment in breast cancer. J Clin Pathol. 2015;68:93-9.

20. Elston CW, Ellis IO. Pathological prognostic factors in breast cancer.I. The value of histological grade in breast cancer: experience from a large study with long term follow up. Histopathology. 1991;19:403-10.

21. Hammond MEH, Hayes DF, Dowsett M, Allred DC, Hagerty KL, Badve S, et al. American Society of Clinical Oncology/College of American Pathologists guideline recommendations for immunohistochemical testing of estrogen and progesterone receptors in breast cancer. J Clin Oncol. 2010;28:2784-95.

22. Page DB, Wen H, Brogi E, Dure D, Ross D, Spinelli KJ, et al. Monosomy 17 in potentially curable HER2-amplified breast cancer: prognostic and predictive impact. Breast Cancer Res Treat. 2018;167:547-54.

23. Krishnamurti U, Hammers JL, Atem FD, Storto PD, Silverman JF. Poor prognostic significance of unamplified chromosome 17 polysomy in invasive breast carcinoma. Mod Pathol. 2009;22:1044-8.

24. Kanda Y. Investigation of the freely available easy-to-use software 'EZR' for medical statistics. Bone Marrow Transpl. 2013;48:452-8.

25. Bursac Z, Gauss CH, Williams DK, Hosmer DW. Purposeful selection of variables in logistic regression. Source Code Biol Med. 2008;3:17.

26. Gianni L, Eiermann W, Semiglazov V, Manikhas A, Lluch A, Tjulandin S, et al. Neoadjuvant chemotherapy with trastuzumab followed by adjuvant trastuzumab versus neoadjuvant chemotherapy alone, in patients with HER2-positive locally advanced breast cancer (the NOAH trial): a randomized controlled superiority trial with a parallel HER2-negative cohort. Lancet. 2010;375:377-84.

27. Gianni L, Pienkowski T, Im YH, Roman L, Tseng LM, Liu MC, et al. Efficacy and safety of neoadjuvant pertuzumab and trastuzumab in women with locally advanced, inflammatory, or early HER2-positive breast cancer (NeoSphere): a randomised multicentre, open-label, phase 2 trial. Lancet Oncol. 2012;13:25-32.

28. Baselga J, Bradbury I, Eidtmann H, Di Cosimo S, de Azambuja E, Aura C, et al. Lapatinib with trastuzumab for HER2-positive early breast cancer (NeoALTTO): a randomised, open-label, multicentre, phase 3 trial. Lancet. 2012;379:633-40.

29. Li AC, Zhao J, Zhao C, Ma Z, Hartage R, Zhang Y, et al. Quantitative digital imaging analysis of HER2 immunohistochemistry predicts the response to anti-HER2 neoadjuvant chemotherapy in HER2-positive breast carcinoma. Breast Cancer Res Treat. 2020;180:321-9. 
30. Press MF, Sauter G, Buyse M, Fourmanoir H, Quinaux E, TsaoWei DD, et al. HER2 gene amplification testing by fluorescent in situ hybridization (FISH): Comparison of the ASCO-College of American Pathologists guidelines with FISH scores used for enrolment in Breast Cancer International Research Group clinical trials. J Clin Oncol. 2016;34:3518-28.

31. Parekh T, Dodwell D, Sharma N, Shaaban AM. Radiological and pathological predictors of response to neoadjuvant chemotherapy in breast cancer: a brief literature review. Pathobiology. 2015;82:124-32.

32. Harbeck N. Insights into biology of luminal her2 vs. Enriched her2 subtypes: therapeutic implications. Breast. 2015;24:S44-8.

33. Miolo G, Muraro E, Martorelli D, Lommbardi D, Scalone S, Spazzapan S, et al. Anthracycline-free neoadjuvant therapy induces pathological complete responses by exploiting immune proficiency in HER2+ breast cancer patients. BMC Cancer. 2014;14:954.

34. Giulianin S, Ciniselli CM, Leonardi E, Polla E, Decarli N, Luchini $\mathrm{C}$, et al. In a cohort of breast cancer screened patients the proportion of HER2 positive cases is lower than that earlier reported and pathological characteristics differ between HER2 $3+$ and HER2 2+/Her2 amplified cases. Virchows Arch. 2016;469:45-50.

35. Tanioka M, Sasaki M, Snimomura A, Fujishima M, Doi M, Matsuura K, et al. Pathological complete response after neoadjuvant chemotherapy in HER2-overexpressing breast cancer according to hormonal receptor status. Breast. 2014;23:466-72.

\section{Affiliations}

Ayaka Katayama $\mathbb{1}^{1,2} \cdot$ Islam M. Miligy ${ }^{1,3} \cdot$ Sho Shiino $0^{1,4} \cdot$ Michael S. Toss $\mathbb{1}^{1} \cdot$ Karim Eldib ${ }^{5} \cdot$ Sasagu Kurozumi ${ }^{6,7} \cdot$ Cecily M. Quinn ${ }^{8}$ - Nahla Badr ${ }^{3,9} \cdot$ Ciara Murray $^{8} \cdot$ Elena Provenzano $\mathbb{1}^{10} \cdot$ Grace Callagy $^{11} \cdot$ Cian Martyn $^{11}$. Rebecca Millican-Slater ${ }^{12}$. Colin Purdie $\mathbb{E}^{13}$ - Dave Purnell ${ }^{14}$ - Sarah E. Pinder $\mathbb{D}^{15} \cdot$ Tetsunari Oyama $^{2}$. Abeer M. Shaaban ${ }^{9} \cdot \operatorname{lan}$ Ellis $\mathbb{1}^{1,5} \cdot$ Andrew H. S. Lee ${ }^{5} \cdot$ Emad A. Rakha ${ }^{1,5}$

1 Nottingham Breast Cancer Research Centre, Division of Cancer and Stem Cells, School of Medicine, Nottingham City Hospital, University of Nottingham, Nottingham, UK

2 Diagnostic Pathology, Gunma University Graduate School of Medicine, Maebashi, Japan

3 Department of Pathology, Faculty of Medicine, Menoufia University, Shebin El-Kom, Egypt

4 Department of Breast Surgery, National Cancer Centre Hospital, Tokyo, Japan

5 Department of Histopathology, Nottingham University Hospitals, Nottingham, UK

6 Department of Breast Surgery, International University of Health and Welfare, Narita, Japan

7 Department of General Surgical Science, Gunma University Graduate School of Medicine, Maebashi, Japan
8 Department of Histopathology, St. Vincent's University Hospital, Dublin, and School of Medicine, University College Dublin, Dublin, Ireland

9 Institute of Cancer and Genomic Sciences, The University of Birmingham, Edgebaston, Birmingham, UK

10 Department of Histopathology, Cambridge University NHS Foundation Trust, Cambridge, UK

11 Discipline of Pathology, School of Medicine, Lambe Institute for Translational Research, NUI Galway, Galway, Ireland

12 Department of Histopathology, St James's University Hospital, Leeds, UK

13 Department of Breast Pathology, Ninewells Hospital and Medical School, Dundee, UK

14 Histopathology department, University Hospitals of Leicester, Leicester, UK

15 Division of Cancer Studies, King's College London, Guy's Hospital, London, UK 